\section{Marked Reduction of Proteinuria after Eradica- tion of Gastric Helicobacter pylori Infection in a Patient with Membranous Nephropathy: Coinci- dental or Associated?}

\author{
Toshiro Sugimoto ${ }^{1}$, Takami Furukawa ${ }^{2}$, Tomohiko Maeda ${ }^{3}$, \\ Mariko Somura ${ }^{1}$, Takashi Uzu ${ }^{1}$ and Atsunori Kashiwagi ${ }^{1}$
}

Key words: Helicobacter pylori, membranous nephropathy

(DOI: 10.2169/internalmedicine.46.0200)

Membranous nephropathy is a common cause of nephrotic syndrome in adults. It is thought to be mainly a primary or idiopathic form; however, it may also be secondary to many other diseases (e.g., infections, drugs, neoplasms, and autoimmune diseases). Here, we describe a patient with membranous nephropathy, whose nephrotic-range proteinuria was markedly reduced after the eradication of gastric Helicobacter pylori infection.

A 34-year-old Japanese woman was admitted because of proteinuria and hematuria. She had no remarkable medical history (i.e., urinary abnormalities or hypertension); however, two months prior to admission she was noted to have urinary abnormalities and iron-deficiency anemia at a routine health examination. She also complained of dyspepsia; thus, gastric endoscopy was performed, which revealed no ulcers, but histologically revealed $H$. pylori infection in the gastric mucosa. On admission, laboratory examination revealed microcytic anemia (hemoglobin, $9.9 \mathrm{~g} / \mathrm{dl}$ ), hypoalbuminemia $(2.7 \mathrm{~g} / \mathrm{dl})$ and nephrotic range-proteinuria $(4.8 \mathrm{~g} /$ day) without active urinary sediments. Renal biopsy revealed normocellular glomeruli with mild capillary wall thickening. Immunofluorescent microscopy disclosed granular deposits of IgG and $\mathrm{C} 3$ on the capillary wall, but no deposits of IgA, IgM, C4, or C1q. Electron microscopy revealed small and discrete electron-dense subepithelial deposits without surrounding glomerular basement membrane reactions, indicating stage I membranous nephropathy. Immunohistochemistry with anti-H. pylori antibodies revealed that the positive staining was found only in the gastric mucosa, but not in the glomeruli from her biopsy specimens (1). Taken together, the fact that the serological studies (e.g., complements, antinuclear antibodies, and hepatitis B/C) yielded negative/normal results, and that she had not taken any medications, her membranous nephropathy was regarded as an idiopathic form.

She showed dyspepsia and iron-deficiency anemia with negative results of fecal occult blood tests, which might have been related to $H$. pylori infection (2); thus, we administrated H. pylori eradication therapy: lansoprazole, $30 \mathrm{mg}$ twice daily, plus amoxicillin, $0.75 \mathrm{~g}$ twice daily, plus clarithromycin, $200 \mathrm{mg}$ twice daily, for 7 days. Four months after this eradication therapy, her gastric symptoms and anemia were improved (hemoglobin, $12.7 \mathrm{~g} / \mathrm{dl}$, Table 1), and her urinary protein excretion had markedly decreased $(0.76$ g/day, Table 1) without any treatments for nephrotic syndrome (e.g., corticosteroids or angiotensin-converting enzyme inhibitors/angiotensin II receptor antagonists, or antiplatelet drugs). Six months after this treatment, the eradication of gastric $H$. pylori infection was confirmed with endoscopic gastric mucosa biopsy. One year after the eradiation of the infection, her proteinuria remains decreased at less than $0.3 \mathrm{~g} /$ day (Table 1 ).

It is well-known that approximately $30 \%$ of the cases of membranous nephropathy resolve spontaneously; however, the occurrence of spontaneous complete remission of this nephropathy within one year from its onset is thought to be rare (less than 5\%) in the untreated patient, as Schieppati et al have reported (3); thus, the eradication of gastric $H$. pylori infection might have contributed to the marked reduction of proteinuria in the present case. The proteinuria associated with membranous nephropathy is thought to be caused by functional impairment resulting from the formation of in situ subepithelial immune deposits and complement activation. Therefore, considerable efforts have been devoted to the identification of antigens involved in human membranous nephropathy, and certain antigens (e.g., hepatitis $\mathrm{B} / \mathrm{C}$, tumor antigens, and DNA-containing materials) have been identified in the immune deposits in patients with so-called secondary membranous nephropathy; however, the pathogenetic role of these antigens in the development of membranous nephropathy has not been confirmed (4). Nagashima et al have reported the presence of $H$. pylori antigens in the glomeruli of membranous nephropathy patients (1). Moriyama et al also found a significantly higher $H$. pylori infection rate in membranous nephropathy patients than in their control group (5). Further, they reported that eradication of $H$. pylori markedly reduced urinary protein excretions in three of four membranous nephropathy patients who were receiving corticosteroid therapy (5). These reports indicate that $H$. pylori infection might play an important role in the development of this disease. In the present case, the immunohistochemistry findings revealed no deposition of $H$.

${ }^{1}$ Department of Internal Medicine, Shiga University of Medical Science, Otsu, ${ }^{2}$ Kusatsu Tokusyukai Hospital, Kusatsu and ${ }^{3}$ Department of Pathology, Yamagata University of School of Medicine, Yamagata

Received for publication March 29, 2007; Accepted for publication May 28, 2007

Correspondence to Dr. Toshiro Sugimoto, toshiro@belle.shiga-med.ac.jp 
Table 1. Laboratory Findings in the Present Case

\begin{tabular}{|c|c|c|c|c|c|c|c|c|}
\hline Variable & $0 \mathrm{mo}$ & $2 \mathrm{mo}$ & $4 \mathrm{mo}$ & $6 \mathrm{mo}$ & $8 \mathrm{mo}$ & $10 \mathrm{mo}$ & $12 \mathrm{mo}$ & reference range \\
\hline Hemoglobin (g/dl) & 9.9 & 10.7 & 12.7 & 13.8 & 13.8 & 14.8 & 15.2 & $11.3-15$ \\
\hline \multicolumn{8}{|l|}{$\left(\mathrm{X} 10^{4} / \mu \mathrm{l}\right)$} & $380-480$ \\
\hline $\operatorname{MCV}(f l)$ & 67 & 66 & 74 & 81 & 86 & 86 & 84 & $83-100$ \\
\hline Total protein (g/dl) & 4.7 & 5.0 & 4.9 & 5.3 & 5.3 & 5.5 & 5.8 & $6.3-8.3$ \\
\hline Albumin (g/dl) & 2.6 & 3.0 & 3.0 & 3.3 & 3.1 & 3.3 & 3.5 & $4-5.2$ \\
\hline Creatinine (mg/dl) & 0.43 & 0.43 & 0.44 & 0.44 & 0.42 & 0.42 & 0.45 & $0.4-0.8$ \\
\hline $\begin{array}{l}\text { Blood urea nitrogen } \\
\qquad(\mathrm{mg} / \mathrm{dl})\end{array}$ & 8 & 10 & 9 & 10 & 9 & 12 & 11 & $8-20$ \\
\hline $\begin{array}{l}\text { Total cholesterol } \\
\text { (mg/dl) }\end{array}$ & 257 & 286 & 241 & 223 & 214 & 199 & 204 & $125-220$ \\
\hline $\begin{array}{l}\text { Urinary total protein } \\
\text { (g/day) }\end{array}$ & 4.8 & 2.0 & 0.76 & 0.50 & 0.72 & 0.39 & 0.24 & \\
\hline Ferritin(ng/ml) & 3.7 & NT & 19.7 & 20.7 & NT & NT & 48.7 & $10-80$ \\
\hline Notes & GF & & & GF & & & & \\
\hline Sodium & $\uparrow \mathrm{E}$ & cati & of $H$ & Iori & & & & \\
\hline
\end{tabular}

mo, months; MCV; mean corpuscular volume; NT, not tested; GF, gastric endoscopy with mucosal biopsy

pylori antigens in the glomeruli. Our patient showed the early stage of membranous nephropathy (Stage I); thus, there is a possibility that enough amounts of $H$. pylori antigens, which can be detected with immunohistochemistry, might not have deposited in our patient's glomeruli. Therefore, the eradication therapy of $H$. pylori could prevent further glomerular deposition of its antigens, which might suppress the progression of membranous nephropathy, resulting in the rapid reduction of proteinuria in our case. Further, there is another possibility that anti-H. pylori antibody might react with podocyte antigens to form immune deposits in situ under the subepithelium, resulting in the development of membranous nephropathy in our case.
To our knowledge, this is the first case report describing that only the eradication of $H$. pylori infection successfully reduced proteinuria in a patient with nephrotic syndrome caused by membranous nephropathy. However, the question remains as to whether the improvement of nephrotic syndrome was due to eradication of this infection, or spontaneous remission of membranous nephropathy. Therefore, further accumulation of clinical studies including case reports is necessary to confirm whether $H$. pylori infection is a secondary aetiological factor in membranous nephropathy and the eradication of this infection might be an affirmative therapy for membranous nephropathy patients with H. pylori infection.

\section{References}

1. Nagashima R, Maeda K, Yuda F, Kudo K, Saitoh M, Takahashi T. Helicobacter pylori antigen in the glomeruli of patients with membranous nephropathy. Virchows Arch 431: 235-239, 1997.

2. DuBois S, Kearney DJ. Iron-deficiency anemia and Helicobacter pylori infection: A review of the evidence. Am J Gastroenterol 100: 453-459, 2005.

3. Schieppati A, Mosconi L, Perna A, et al. Prognosis of untreated patients with idiopathic membranous nephropathy. N Engl J Med
29: 85-89, 1993.

4. Ronco P, Debiec H. Molecular pathomechanisms of membranous nephropathy: from Heymann nephritis to alloimmunization. J Am Soc Nephrol 16: 1205-1213, 2005.

5. Moriyama T, Kaneko T, Fujii M, Tsubakihara Y, Kawano S, Imai E. High prevalence of Helicobacter pylori infection in Japanese patients with membranous nephropathy. Aliment Pharmacol Ther suppl 4: 189-193, 2006.

(C) 2007 The Japanese Society of Internal Medicine http://www.naika.or.jp/imindex.html 